\title{
Bioequivalence between Generic Rasagiline (As Tartrate) and Rasagiline (As Mesylate)
}

\author{
Van Rijswick YGJ, Quesada MJ and Van Os SHG \\ Synthon BV, 6503 GN Nijmegen, Netherlands
}

*Corresponding author: Van Rijswick YGJ, Synthon BV, 6503 GN Nijmegen, The Netherlands, Fax: +31 (0)24 37278 75, Tel: +31 (0)24 37277 00, E-mail: Yvonne.vanRijswick@synthon.com

Citation: Van Rijswick YGJ, Quesada MJ, Van Os SHG (2016) Bioequivalence between Generic Rasagiline (As Tartrate) and Rasagiline (As Mesylate). J Bioequiv 2(1): 104. doi: 10.15744/2575-551X.2.104

Received Date: August 12, 2016 Accepted Date: November 11, 2016 Published Date: November 14, 2016

\begin{abstract}
Rasagiline is a selective irreversible monoamine oxidase type B (MAO-B) inhibitor and indicated for the treatment of idiopathic Parkinson's disease (PD).

Objective: The objective of the study was to compare bioavailability of $1 \mathrm{mg}$ rasagiline (as tartrate) immediate release tablets and Azilect $^{\bullet} 1 \mathrm{mg}$ immediate release tablets.

Methods: A bioequivalence study with generic $1 \mathrm{mg}$ rasagiline (as tartrate) immediate release tablets versus $1 \mathrm{mg}^{\text {Azilect }} \mathrm{immediate}^{\circledR}$ release tablets was performed in healthy volunteers under fasting conditions.

Results: The results show that the $90 \%$ confidence intervals for the geometric means ratio of the formulations were within the acceptance range of 80.00 to $125.00 \%$.

Conclusion: Bioequivalence between the $1 \mathrm{mg}$ generic rasagiline (as tartrate) immediate release tablets and the $1 \mathrm{mg}$ Azilect ${ }^{\oplus}$ immediate release tablets has been proven in this study.
\end{abstract}

Keywords: Rasagiline; Bioequivalence; Pharmacokinetics; Mannitol; Tartrate; Mesylate; Azilect

\section{Introduction}

Rasagiline is marketed by TEVA Pharmaceutical Industries as Azilect ${ }^{\circledR}$ and is approved in the EU for the treatment of idiopathic Parkinson's disease (PD) as monotherapy (without levodopa) or as adjunctive therapy (with levodopa) in patients with end of dose fluctuations. Rasagiline is a selective irreversible monoamine oxidase type B (MAO-B) inhibitor. MAO inhibitors decrease the oxidative deamination of dopamine, both endogenous dopamine as well as dopamine produced from exogenous levodopa. The result is that dopamine levels are increased and dopaminergic function is restored resulting in improvement of motorsymptoms [1-4].

A generic immediate release tablet containing $1 \mathrm{mg}$ of rasagiline (as tartrate salt) was developed and the necessity for a study to register the generic rasagiline in the European Union was assessed. Bioequivalence studies are generally accepted as the most appropriate proof to establish therapeutic equivalence between a product used in the clinical studies and a new product with the same active substance. It may be inferred that an equivalent plasma concentration profile will result in essentially similar concentrations at the site of action. Based on this assumption, an equivalent rate and extent of absorption will predict an essentially similar effect and safety profile. Therefore, when two pharmaceutical equivalents or alternatives are bioequivalent after administration in the same molar dose, their effects, with respect to both efficacy and safety, will be essentially the same [5-7].

In the European Union, the biopharmaceutics classification system (BCS) - a scientific framework for classifying a drug substance based on its aqueous solubility and intestinal permeability - can be used to apply for waivers of in vivo bioequivalence studies for immediate release solid oral dosage forms. This is generally called a biowaiver. Sponsors may request for a biowaiver for immediate release solid oral dosage forms of highly soluble and highly permeable drugs (Class I) that exhibit very rapid or similarly rapid in vitro dissolution ( $85 \%$ dissolved in $30 \mathrm{~min}$ ) or for highly soluble low permeable drugs (Class III) that exhibit very rapid dissolution ( $>85 \%$ dissolved in $15 \mathrm{~min}$ ). The scientific rationale is that BCS Class I and III products may behave in vivo like an oral solution with the membrane permeability being expected to be the rate-limiting step in drug absorption. Therefore, absorption kinetics from the gastrointestinal tract would be governed by drug biopharmaceutic factors and physiologic factors, rather than by formulation factors, given that excipients have no relevant effects on gastrointestinal transit and permeability of drug $[5,8]$. 
Rasagiline is a highly soluble low permeable drug and therefore classifies as a BCS class III product. Bioequivalence between the generic product and the product for which clinical safety and efficacy studies were done by the Originator can be assumed when the test product and reference product both show very fast dissolution profiles unless there is a quantitative and/or qualitative difference in excipients that might affect bioavailability [5,8]. The originator product, Azilect ${ }^{\oplus}$, contains mannitol whereas the generic rasagiline tablets do not. Mannitol is one of the excipients, explicitely mentioned in the EMA guideline, known to possibly affect the gastrointestinal motility [5,9-11]. The quantities of mannitol affecting gastrointestinal motility are unknown and therefore a bioequivalence study was deemed necessary to register the generic rasagiline tablets.

The objective of the study was to compare the bioavailability of the generic rasagiline (test) tablets and the Azilect ${ }^{\circledR}$ tablets (reference) in healthy volunteers under fasting conditions and to assess bioequivalence.

\section{Materials and Methods}

\section{Dissolution testing}

The dissolution of 12 rasagiline test and reference tablets was tested using USP II in vessels containing $900 \mathrm{ml} 0.1 \mathrm{~N} \mathrm{HCL}$, acetate buffer $\mathrm{pH} 4.5$ and phosphate buffer $\mathrm{pH} 6.8$ at $37^{\circ} \mathrm{C}$. The amount of dissolved rasagiline was measured at 5, 10, 15, 20, 30 and 45 minutes.

\section{Bioequivalence study}

The bioequivalence study was a randomized, two-treatment, four-period, two-sequence, single-dose, replicate-crossover bioequivalence study comparing rasagiline (as tartrate) $1 \mathrm{mg}$ tablets (test) to rasagiline (as mesylate) $1 \mathrm{mg}$ tablets (Azilect ${ }^{\oplus}$, reference) in healthy, adult male volunteers under fasting conditions.

A total of thirty volunteers were enrolled in the study. All volunteers were aged between 18 to 45 years with a body mass index in a range of $18.5 \mathrm{~kg} / \mathrm{m}^{2}$ to $24.9 \mathrm{~kg} / \mathrm{m}^{2}$, provided written informed consent and were willing to follow the protocol requirements. The study protocol was approved by the DCGI and Ethical Committee before study start and was conducted according to the Declaration of Helsinki, ICH GCP guidelines EMA guidelines, ICMR and CDSCO guidelines.

The volunteers were confined within the facility from at least 10.50 hours before dosing until 24.00 hours post-dose in each study period. After an overnight fast of at least 8 hours, all volunteers were dosed with the study drug-a single oral dose of test product or reference product according to the randomization schedule - in sitting position with $200 \mathrm{~mL}$ of water at ambient temperature in each study period.

Safety measurements included monitoring of adverse events, physical examination, well-being assessment, vital-signs assessment, 12 lead ECG and clinical laboratory tests.

During each study period, a total of 17 blood samples $(5 \mathrm{~mL}$ each) were collected from the subjects in heparin vacutainers at different time points: pre-dose (collected within $1 \mathrm{hr}$ prior to dosing), 0.083, 0.16, 0.33, 0.50, 0.67, 0.83, 1.00, 1.50, 2.00, 2.50, 3.00, $4.00,6.00,8.00,12.00$ and 24.00 hours post-dose. Analysis of plasma concentrations of rasagiline was done by a validated LC-MS/ MS analytical method.

Assessment of bioequivalence was done by comparing pharmacokinetic parameters of the rasagiline (as tartrate) $1 \mathrm{mg}$ tablets with the reference product Azilect ${ }^{\oplus} 1 \mathrm{mg}$ tablets. For the primary endpoints ANOVA was performed on Ln- transformed pharmacokinetic parameters $\mathrm{C}_{\max }$ and $\mathrm{AUC}_{0-\mathrm{t}}$ for rasagiline. The $90 \%$ confidence interval was constructed for the ratio of geometric least square means of the test and reference product, obtained from the Ln-transformed pharmacokinetic parameters $C_{\max }$ and AUC ${ }_{0-t}$ All pharmacokinetics and statistical analysis have been performed by the use of SAS version 9.2. Bioequivalence was concluded if the $90 \%$ confidence intervals of the ratio of least square means (test/reference) of $\mathrm{C}_{\max }$ and $\mathrm{AUC}_{0-\mathrm{t}}$ were within the acceptance range of 80.00-125.00\%. If it was demonstrated that the intra-subject variability for $\mathrm{C}_{\max }$ for the reference product was $>30 \%$, then widened acceptance criteria for $\mathrm{C}_{\max }$ could be considered using reference scaled average bioequivalence with a maximum of $69.84-143.19 \%$.

\section{Results}

\section{Assay}

The assay of the tablets used in the study was $100.0 \%$ for Azilect ${ }^{\circledR}$ containing $1 \mathrm{mg}$ rasagiline (as mesylate) and 101.9\% for the generic rasagiline tablets containing $1 \mathrm{mg}$ rasagiline (as tartrate).

\section{Dissolution testing}

Both Azilect ${ }^{\oplus}$ as well as generic rasagiline showed very fast dissolution with $>90 \%$ dissolved within 15 minutes at $0.1 \mathrm{~N} \mathrm{HCl}$, $\mathrm{pH} 4.5$ and 6.8 as can be seen in Figure 1. 


\section{$0.1 \mathrm{~N} \mathrm{HCL}$}

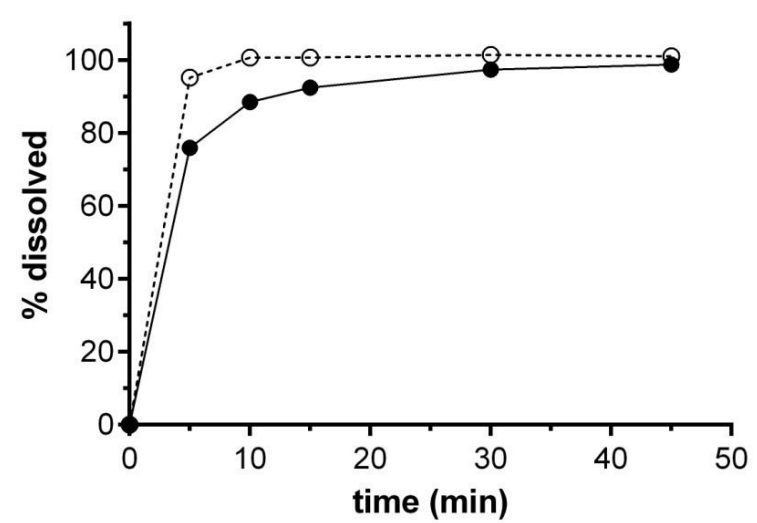

(b) acetate buffer $\mathrm{pH} 4.5$

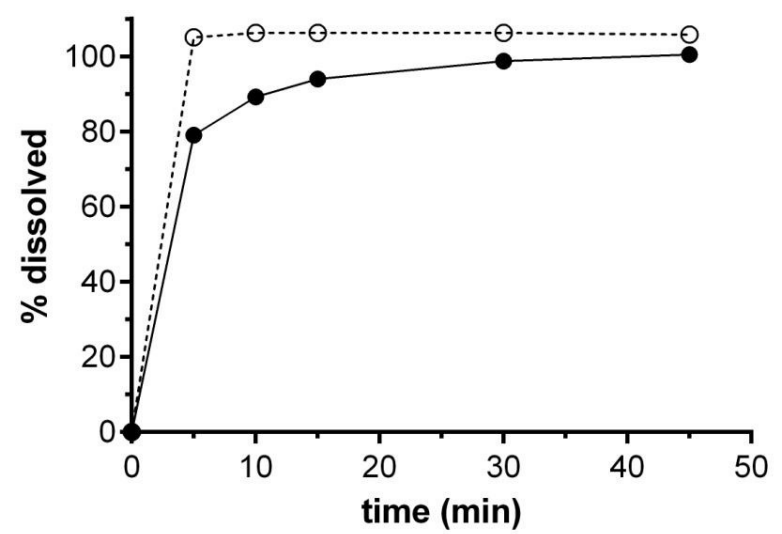

(c) $\quad$ phosphate buffer $\mathrm{pH} 6.8$

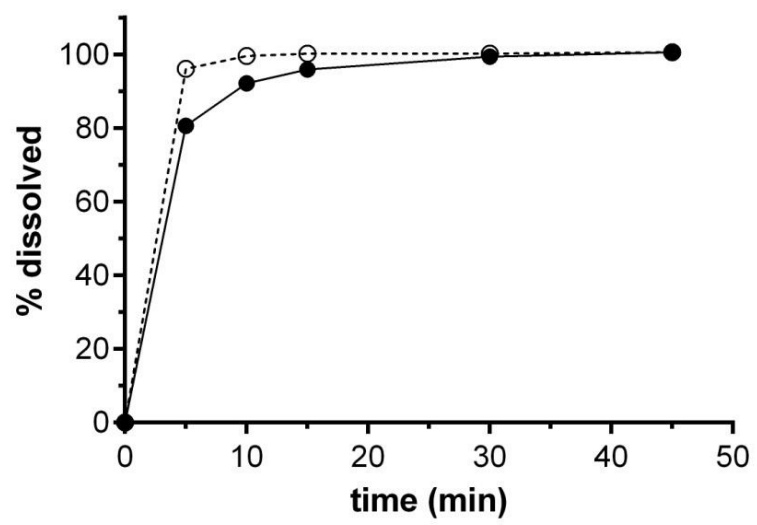

Figure 1: Dissolution profiles of generic rasagiline (closed marks) and Azilect ${ }^{\oplus}$ (open marks) at a) $\left.0.1 \mathrm{~N} \mathrm{HCL}, \mathrm{b}\right) \mathrm{pH} 4.5$ and c) pH 6.8

\section{Bioequivalence study}

Two subjects dropped out as they did not report to the study center for period II, III and IV, one subject dropped out as the subject did not report to the study center for period II and III and two subjects were withdrawn due to an adverse event after dosing in Period I. Therefore, a total of twenty five subjects completed the clinical phase of the study successfully and received both the test and reference products twice. Twenty six subjects received at least once one test product and once the reference product and were considered for pharmacokinetic analysis.

A total of six adverse events were reported during the clinical phase of the study of which one adverse event was observed in a subject who received test product and two adverse events were observed in two subjects that received reference product. These 
three adverse events were judged by the Principle Investigator to be expected to be related to the study medication. Three adverse events were observed during the post-study evaluation. One subject had a high eosinophil count, one was low in hemoglobin and one had a high white blood cell count. The Principal Investigator judged these three adverse events to be unexpected and unrelated to the study medication. All the adverse events were moderate to mild in severity. No serious adverse events were observed.

The mean plasma-concentration versus time profiles are presented in Figure 2
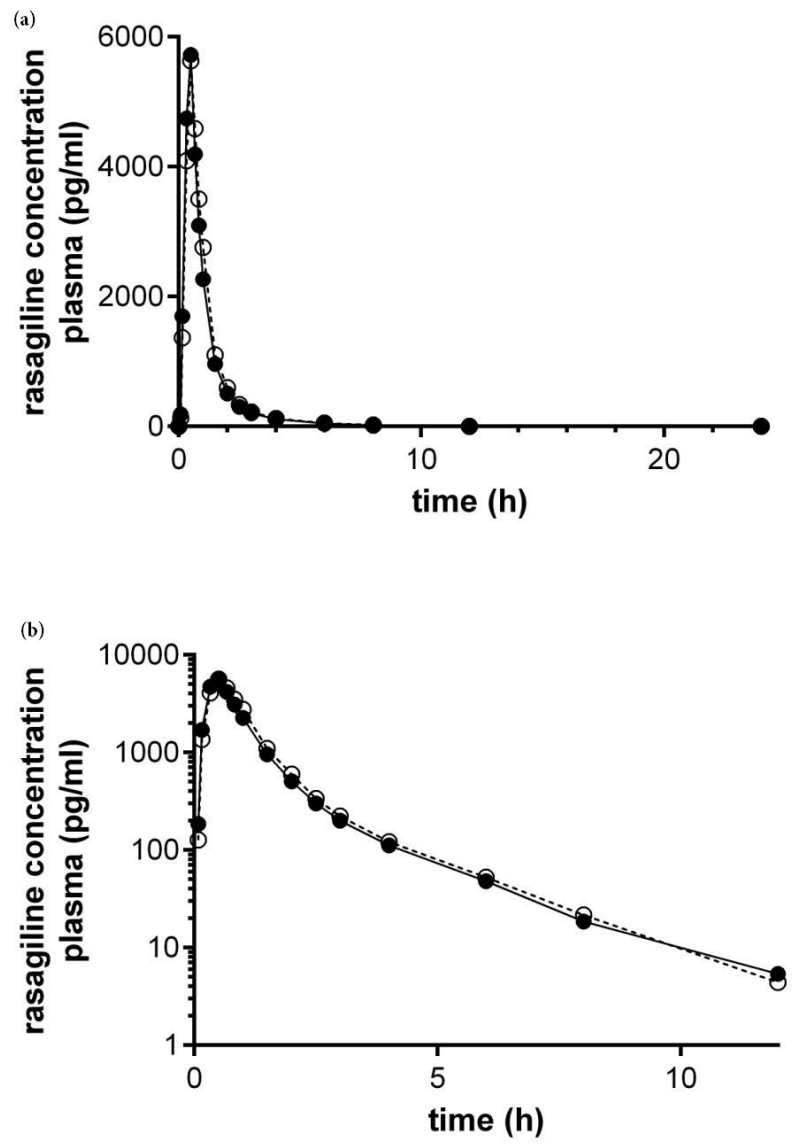

Figure 2: Plasma concentration versus time profiles of generic rasagiline (closed marks) and Azilect ${ }^{\oplus}$ (open marks) at a) linear scale and b) logarithmic scale

The intra-subject variability for $\mathrm{C}_{\max }$ of the reference product was found to be $34.71 \%$ and hence widened acceptance criteria (76.8-130.2\%) could be applied for $\mathrm{C}_{\max }$. The mean of $\mathrm{AUC}_{0-\mathrm{t}} / \mathrm{AUC}_{0 \text {-inf }}$ ratio was $97.19 \%$ for the test formulation and $97.24 \%$ for the reference formulation.

The ratio of geometric least square means of the test and reference product with the corresponding $90 \%$ confidence intervals of the ln-transformed parameters are summarized in Table 1. The means of $\mathrm{t}_{\max }, \mathrm{K}_{\mathrm{el}}$ and $\mathrm{t}_{1 / 2}$ for both formulations are provided in Table 2.

\begin{tabular}{|c|c|c|c|c|c|}
\hline \multirow{2}{*}{ Parameters } & \multicolumn{2}{|c|}{${ }^{*}$ Geometric mean (pg/ml) } & \% Ratio & \multicolumn{2}{c|}{$\begin{array}{c}\text { 90\% Confidence Interval for } \\
\text { Ln-transformed data }\end{array}$} \\
\cline { 2 - 6 } & Generic rasagiline & Azilect $^{\circledR}$ & T/R & Lower Limit & Upper Limit \\
\hline $\mathrm{C}_{\max }$ & 5943.59 & 6370.66 & 93.30 & 84.54 & 102.97 \\
\hline $\mathrm{AUC}_{0-\mathrm{t}}$ & 4756.49 & 4961.47 & 95.87 & 90.60 & 101.45 \\
\hline
\end{tabular}

${ }^{*}$ Geometric mean was taken as the antilog (exponential) of the least square mean of the ln-transformed data Table 1: Geometric means and 90\% Confidence Interval for generic rasagiline versus Azilect ${ }^{\oplus}(\mathrm{N}=26)$

\begin{tabular}{|c|c|c|c|}
\hline & $\mathbf{t}_{\mathbf{1} / 2}( \pm \mathbf{S D})(\mathbf{h})$ & $\mathbf{T}_{\max }(\mathbf{h})$ & $\mathbf{K}_{\mathrm{el}}\left(\mathbf{h}^{-1}\right)$ \\
\hline Generic rasagiline $^{-1}$ & $1.59 \pm 0.98$ & $0.52 \pm 0.21$ & $0.63 \pm 0.38$ \\
\hline Azilect $^{\circ}$ & $1.58 \pm 0.95$ & $0.56 \pm 0.27$ & $0.63 \pm 0.39$ \\
\hline
\end{tabular}

Table 2: Average $\mathrm{T}_{\max }, \mathrm{t}_{1 / 2}$ and $\mathrm{K}_{\mathrm{el}}$ of generic rasagiline and Azilect ${ }^{\oplus}$ 


\section{Discussion}

The results show that the $90 \%$ confidence intervals for the geometric means ratio of the generic rasagiline (as tartrate) 1 mg tablets and the Azilect ${ }^{\oplus} 1 \mathrm{mg}$ tablets were within the acceptance range of 80.00 to $125.00 \%$ for rasagiline.

Even though the generic rasagiline and Azilect ${ }^{\oplus}$ contain different salt forms, the therapeutic activity of rasagiline resides with the active substance rasagiline and not with the salt form. Therefore, when a rasagiline tablet is taken, the rasagiline will first be dissolved in the fluids of the stomach before the rasagiline is taken up by the human body. After uptake, exactly the same rasagiline molecule is present in the human body for the therapeutic effect, independent of the mesylate or tartarte origin. Both the generic rasagiline as well as Azilect ${ }^{\oplus}$ showed similarity in dissolution with both having very fast dissolution profiles. Additionally, the bioequivalence study showed that the $90 \%$ confidence intervals for the geometric means ratio of the generic rasagiline (as tartrate) $1 \mathrm{mg}$ tablets and the Azilect ${ }^{\oplus} \mathrm{mg}$ tablets are contained within the acceptance range of 80.00 to $125.00 \%$ for rasagiline. Therefore, it can be concluded that both formulations containing different salts have equivalent bioavailability of rasagiline.

Another difference between the generic rasagiline formulation and Azilect ${ }^{\circledast}$ is that Azilect ${ }^{\circledast}$ contains mannitol, whereas the generic rasagiline does not contain mannitol. According to the EMA guideline on bioequivalence, a BCS-based biowaiver is not acceptable for any BCS-class I or III drug when there is any qualitative and/or quantitative difference in any excipients known to affect bioavailability [4]. The guideline explicitly mentions mannitol as an example of excipients known to possibly affect bioavailability. Adkins et al. previously demonstrated that doses of mannitol between $0.755 \mathrm{~g}$ and $2.264 \mathrm{~g}$ can accelerate small-intestinal transit time and thereby could cause impaired absorption [1,3]. However, the smallest amount of mannitol tested, $0.755 \mathrm{~g}$, is still relatively high for the pharmaceutical industry. It is unknown if a lower amount of mannitol also accelerates small-intestinal transit time. If mannitol would affect the bioavaibility in the Azilect ${ }^{\circledast}$ formulation in comparison to the test formulation of rasagiline, one would expect a point estimate in the $\mathrm{T} / \mathrm{R}$ ratio higher than $100 \%$. Although the change in mannitol was obviously not the only change between the formulations, and therefore the effect of mannitol could theoretically have been counteracted, a difference in mannitol of $159 \mathrm{mg}$ did not seem to affect the bioavailability for rasagiline.

\section{Conclusion}

This study has demonstrated that the generic rasagiline and Azilect ${ }^{\circledR}$ show an equivalent rate and extent of absorption. Therefore, these two rasagaline formulations are bioequivalent after administration. Because the generic rasagiline and Azilect ${ }^{\oplus}$ behave the same in terms of uptake, distribution, metabolism and excretion, the generic rasagline can be considered as having the same therapeutic effect and safety profile as Azilect ${ }^{\oplus}$, given that the posology of administration is followed.

\section{Acknowledgement}

We would like to thank the staff of Accutest for the conduct, bioanalysis and PK of the study.

\section{References}

1. Parkinson Study Group (2002) A controlled trial of rasagiline in early Parkinson disease: the TEMPO Study. Arch Neurol 59: 1937-43.

2. Parkinson Study Group (2004) A controlled, randomized, delayed-start study of rasagiline in early Parkinson disease. Arch Neurol 61: 561-6.

3. Parkinson Study Group (2005) A randomized placebo-controlled trial of rasagiline in levodopa-treated patients with Parkinson disease and motor fluctuations: the PRESTO study. Arch Neurol 62: 241-8.

4. Thebault JJ, Guillaume M, Levy R (2004) Tolerability, safety, pharmacodynamics, and pharmacokinetics of rasagiline: a potent, selective, and irreversible monoamine oxidase type B inhibitor. Pharmacotherapy 24: 1295-305.

5. CPMP (2010) Guideline on the investigation of bioequivalence (CPMP/EWP/QWP/1401/98 Rev.1/Corr ${ }^{* *}$ ).

6. CPMP (2000) Note for Guidance on the Investigation of Bioavailability and Bioequivalence. PMP/EWP/QWP/1401/98.

7. FDA (2005) Title 21 Code of Federal Regulation: Bioavailability and Bioequivalence requirements. 21 CFR 320. 21.

8. Yu LX, Amidon GL, Polli JE, Zhao H, Mehta MU, et al. (2002) Biopharmaceutics classification system: the scientific basis for biowaiver extensions. Pharm Res 19: 921-5.

9. Adkin DA, Davis SS, Sparrow RA, Huckle PD, Phillips AJ, et al. (1995) The effect of different concentrations of mannitol in solution on small intestinal transit: implications for drug absorption. Pharm Res 12: 393-6.

10. Adkin DA, Davis SS, Sparrow RA, Huckle PD, Phillips AJ, et al. (1995) The effects of pharmaceutical excipients on small intestinal transit. Br J Clin Pharmacol 39: $381-7$.

11. Adkin DA, Davis SS, Sparrow RA, Huckle PD, Wilding IR (1995) The effect of mannitol on the oral bioavailability of cimetidine. J Pharm Sci 84: 1405-9. 


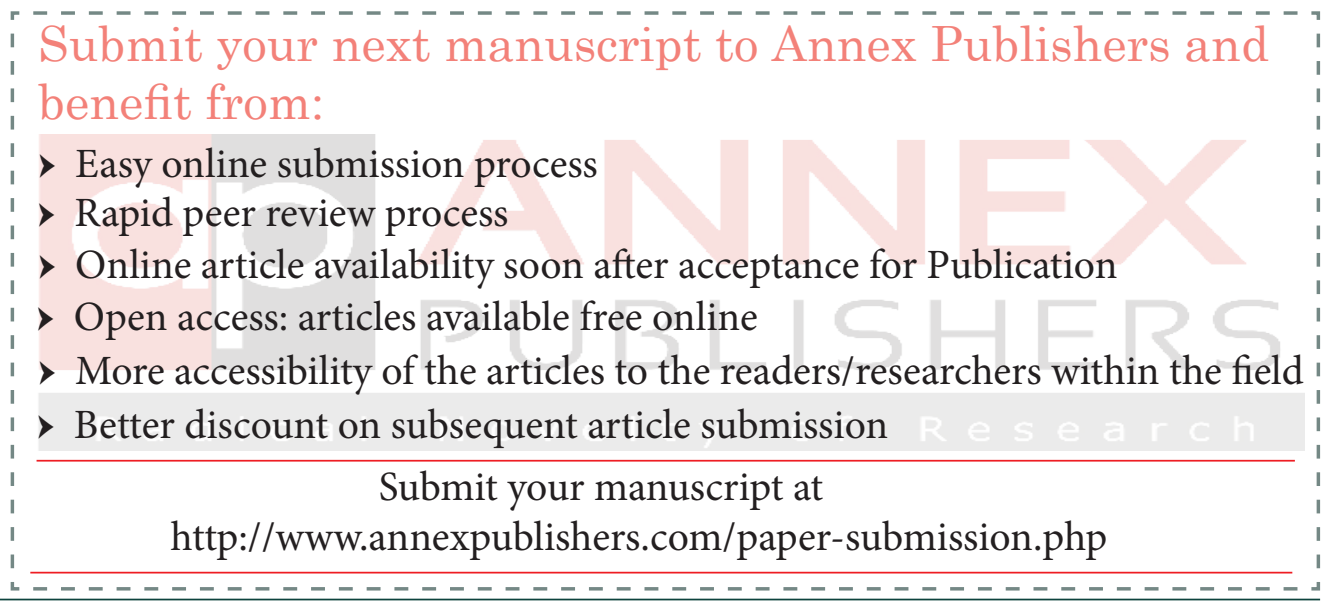

